\title{
Analysis of a planetary gear modelled with a contour graph considering the decision making complexity of game-tree structures
}

\author{
Adam Deptuła ${ }^{1, *}$, Józef Drewniak ${ }^{2}$, and Marian A. Partyka ${ }^{1}$ \\ ${ }^{1}$ Opole University of Technology, Faculty of Production Engineering and Logistics, Institute of Processes and Products \\ Innovation, Ozimska 75, 45-370 Opole, Poland, \\ ${ }^{2}$ University of Bielsko-Biala, Faculty of Machine Building and Computer Science, Department of Mechanical Engineering \\ Fundamentals, Willowa 2, 43-309 Bielsko-Biała, Poland
}

\begin{abstract}
Analysis and synthesis of mechanisms is one of the fundamental tasks of engineering. Mechanisms can suffer from errors due to versatile reasons. Graph-based methods of analysis and synthesis of planetary gears constitute an alternative method for checking their correctness. Previous applications of the graph theory concerned modelling gears for dynamic analysis, kinematic analysis, synthesis, structural analysis, gearshift optimization and automatic design based on socalled graph grammars. Some tasks may be performed only with the methods resulting from the graph theory, e.g. enumeration of structural solutions. The contour plot method consists in distinguishing a series of consecutive rigid units of the analysed mechanism, forming a closed loop (so-called contour). At a later stage, it is possible to analyze the obtained contour graph as a directed graph of dependence. This work presents an example of the application of game-tree structures in describing the contour graph of a planetary gear. In addition, complex parametric tree structures are included.
\end{abstract}

\section{Introduction}

Engineering practice requires correct evaluation of the mathematical model describing a given system with certain variables. A proper mathematical model contains a group of functions joining different variables and describing connections between quantities in the system. Decision tables [1-3] and logical functions [3-4] can be applied in simulation of machine systems, for example described by ordinary or partial differential equations. It results from the fact that the occurring nonlinear elements can be divided into the finite number of linear elements (parts), and as a consequence we obtain a linear simulation system from the primarily single nonlinear system. Analysis and synthesis of mechanisms can be performed by means of versatile methods. These tasks can suffer from human errors. It is therefore reasonable to employ some alternative methods which will allow for comparison of results and for detection of almost unavoidable mistakes. The graph-based methods deliver such an alternative approach to modelling of a wide class of mechanical systems. There have also been other attempts to model planetary gears via diagrams e.g. Wolf's pictograms [5], but those methods did not further evolve due to lack of generalization and lack of connections with other branches of mathematics $[6,7]$. Furthermore, a method based on the signal flow graph theory for modelling gears $[8,9]$ has not been too frequently used. On the contrary, the graph based methods have been independently and intensively developed in recent years all over the world (see e.g. [10]). The superiority of the graph-based modelling of mechanical systems consists in the fact that a graph is inseparably connected with other algebraic structures like e.g. subgraphs (e.g. trees, cycles, cliques, paths), dual graph, matrices, vector spacer of cuts and cycles, structural numbers and matroids as well as algorithms connected with these structures. By interpreting the mechanical knowledge In terms of graphs, we solve mechanical problems via graph models [11]. Versatile linear graph models were considered by Tsai [12] but Hsu's graph models of gears [13] are utilized in the following considerations. In the case of analysis of car gearboxes, the graph-based methodology is also efficient when incorporating additional transformations of a basic graph according to a particular gear driver [5]. An introductory comparison of the graph and contour methods for analysis of gears was done by the authors in $[5,10]$.

The occurring constructional parameters influence courses of unknown functions dependent on time. A traditional analysis, such as Input $\rightarrow$ Output and Output $\rightarrow$ Input, of a given system with the dependence graph method enables obtaining the vertex groups of the following properties:

- elements inside the given group contain many information connections,

Corresponding author: a.deptula@po.opole.pl 
- particular groups have few reciprocal information connections.

In this way we obtain constructional recommendations for obtaining multiple solutions from the dependence graph, so the selection of suitable subsolutions can be done with use of tree classifiers from the neuron networks. Let us consider a different approach: the translation of the dependence digraph into the tree game structure [14, 15]. Constructional and service parameters may be joined in different mathematical ways, it is necessary to determine precise decomposition of the dependence graph in order to define decision variables. Such procedure may determine the area of feasible solutions allowing to select a proper optimization procedure.

\section{The dependence graph for tree game structures}

The equations of dynamics can be used for determination of mutual connections of all the functions dependent on time. As a result of notation and decomposition of the dependence graph of those functions we obtain the groups of distribution which describe properties of successive subsystems of the considered machine system and a set of suitable constructional and service parameters.

An oriented graph can be defined as an ordered pair of sets. The graph vertices are included in the first one, whereas graph edges, which is an ordered pair of vertices, are included in the other (Fig. 1) $[14,15]$.

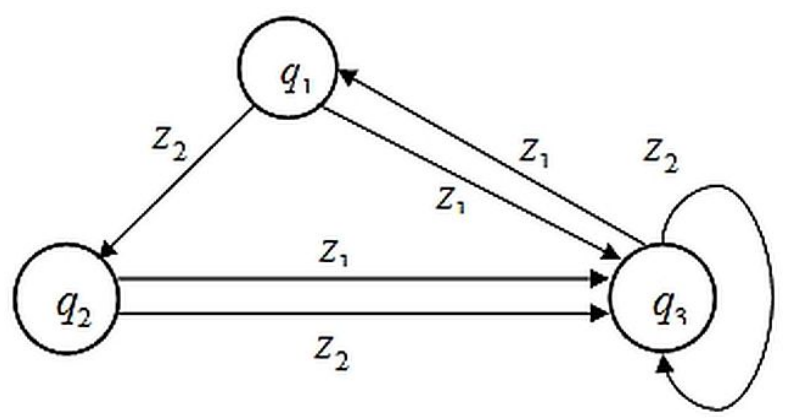

Fig. 1. An oriented game graph.

The oriented game graph shown in Fig. 1 is composed of a set of vertices $Q$ :

$$
Q=\left\{q_{1}, q_{2}, q_{3}\right\}
$$

and of a set of edges $Z$, which is an ordered pair of vertices:

$$
Z=\left\{z_{1}, z_{2}\right\}
$$

As a result of a graph distribution from the chosen vertex, a tree structure with cycles is obtained in the first step and then, a general game tree structure is obtained. Each of them has an appropriate analytical formulation $G_{i}^{+}$and $G_{i}^{++}$.
A game tree structure is a part of a systematic research method. A start vertex $q_{i}$ is chosen in the first step. Acting in accordance with the algorithm and assuming that the start vertex is $q_{1}$ it is possible to transform the oriented dependence graph presented in Figure 1 into an analytical formulation $G_{q_{1}}^{+}$, and then we obtain the following formulation as a result of the operation (3).

$$
G_{1}^{+}=\left({ }^{0} q_{1}\left({ }^{1} z_{1} q_{3}\left({ }^{2} z_{1} q_{1}, z_{2} q_{3}\right)^{2}, z_{2} q_{2}\left({ }^{2} z_{1} q_{3}, z_{2} q_{3}\right)^{2}\right)^{1}\right)^{0}(3)
$$

The next step is to obtain a tree structure that plays a parametrically defined expression $[14,15](4)$ :

$$
\begin{aligned}
& G_{1}^{++}=\left({ } ^ { 0 } q _ { 1 } \left({ }^{1} z_{1} q_{3}\left({ }^{2} z_{1} q_{1}^{1}, z_{2} q_{3}^{1}\right)^{2}, z_{2} q_{2}\left({ }^{2} z_{1} q_{3}\left({ }^{3} z_{1} q_{1}^{2}, z_{2} q_{3}^{1}\right)^{3},\right.\right.\right. \\
& \left.\left.\left.z_{2} q_{3}\left({ }^{3} z_{1} q_{1}^{2}, z_{2} q_{3}^{1}\right)^{3}\right)^{2}\right)^{1}\right)^{0}
\end{aligned}
$$

It is possible to return to an earlier vertex and even to a start vertex from an appropriate end vertex; we obtain a game tree structure presented in Fig. 2.

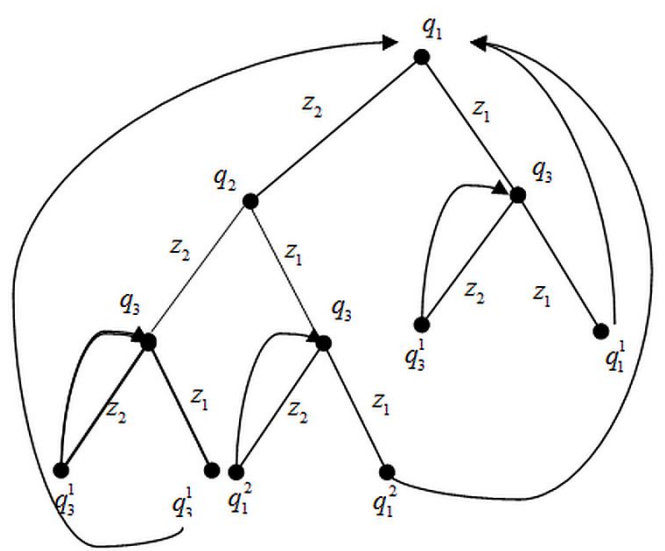

Fig. 2. Tree structure with cycles and the initial vertex $q_{1}$.

When we change cycles in the structure shown in Fig. 2 into decisions and/or expressions we obtain a game tree structure presented in Fig. 3.

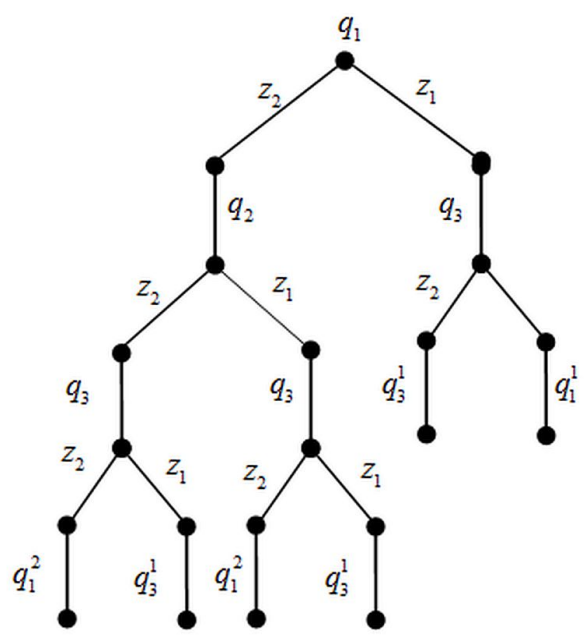

Fig. 3. Tree game structures from the initial vertex $q_{1}$. 
The contour graph distribution from any vertex in the first stage leads to a tree structure with cycles, and next to a general tree game structure. Each structure has a proper analytic notation $G_{i}^{++}$- where $i$ - is a vertex, from which the graph decomposition started, determining a way of transition from the dependence graph to the tree structure.

\section{Graph based model of a planetary gear}

The general idea of the graph-based modelling of mechanical systems consists in the following steps [16]:

- discretisation of a mechanical system. This means that appropriate simplifications have to be made. Some aspects are omitted. Some structural elements are considered as essential and they are interpreted as graph vertices. Some connections or relationships between these elements are abstracted. They are represented via graph edges, usually a system of weights can be assumed to the edges or vertices and edges,

- assignment of the graph to the mechanism (especially a planetary gear) based upon special rules. There are several different rules depending on the object of modelling and problems solved via the graph based method,

- derivation of special subgraphs, e.g. f-cycles or contours; these subgraphs can be singled out based upon the graph-theoretical algorithms, which causes that the approach is simple and algorithmic,

- listing the codes of these graph elements; the encoding rules are clearly defined what allows for avoidance of mistakes,

- generation of a system of equations in an algorithmic way using the codes; these codes allow for management (assignment) of indicators of variables existing in the considered equations in a straightforward manner,

- solution of the obtained system in a chosen algebraic manner to obtain desired angular velocities, ratios, forces, accelerations etc.

A similar routine may be formulated for the reverse order of activities, i.e. synthesis: going from the graph generation towards the creation of a gear functional structure [12].

The contour method of modelling of a mechanical system consists in creation of a special graph enclosing the contours, i.e. closed circles built of arrows connecting the vertices representing the elements of the system. Planetary gears are mechanisms consisting inter alia of geared wheels, in which at least the symmetry axis of one wheel encircles the main symmetry axis of the system. The wheel, which is going round, is called a planetary wheel or even just a planet or a satellite gear $[16,17,18]$. In case when all three basic gear parts are movable, then a planetary gear has two degrees of freedom and there is a demand for driving two different basic elements. In such a case, a system serves as a differential gear. The considered planetary gear is presented in Fig. 4.

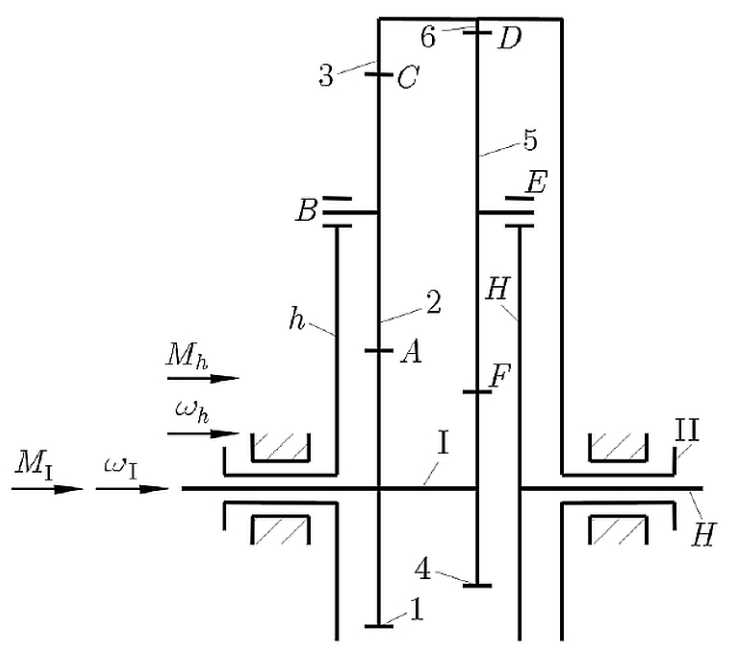

Fig. 4. Functional scheme of an exemplary planetary gear: $1,2, \ldots, 6$ sun wheels, wheels with internal toothings and planets; $h, H-$ arms; $A, B, \ldots, F$-characteristic points, $M$-angular velocities and torques.

A special planetary gear is considered. It encloses an internal closed loop formed by wheels and planets. The mobility W (DOF, degree of freedom) for the considered mechanism, i.e. planetary gear, can be calculated from the following formula $[5,6,7,19](5)$ :

$$
W=3 n-2 c 5-c 4=18-12-4=2
$$

where: $n$ - number of links (movable elements), c5 number of full joints (one degree of freedom), e.g. rotational type; $c 4$ - number of half joints, e.g. meshing type; moreover $c 5$ and $c 4$ are equal to the total number of edges and diagonals of a polygon $(c 5=6)$ and the number of stripped edges $(c 4=4)$, respectively. We also have $\mathrm{n}=6$, i.e. the number of graph vertices - in the case of linear graph representation of a planetary gear.

For the considered gear, the following data for teeth numbers and the module are assumed: $z 1=15 ; z 2=24 ; z 3$ $=63(-63) ; z 4=18 ; z 5=21$ and $z 6=60(-60), m=2$ $\mathrm{mm}$, where negative values of the teeth numbers are considered for the internal gearing in the case of the Willis method and one common module for all meshings was assumed.

\subsection{Contour based model of the planetary gear}

The contour method of modelling a mechanical system consists in creating a special graph enclosing the contours, i.e. closed circles built of arrows connecting the vertices representing the elements of the system [20, 21]. The contour graph of the gear presented in Fig. 4 is shown in Fig. 5. 


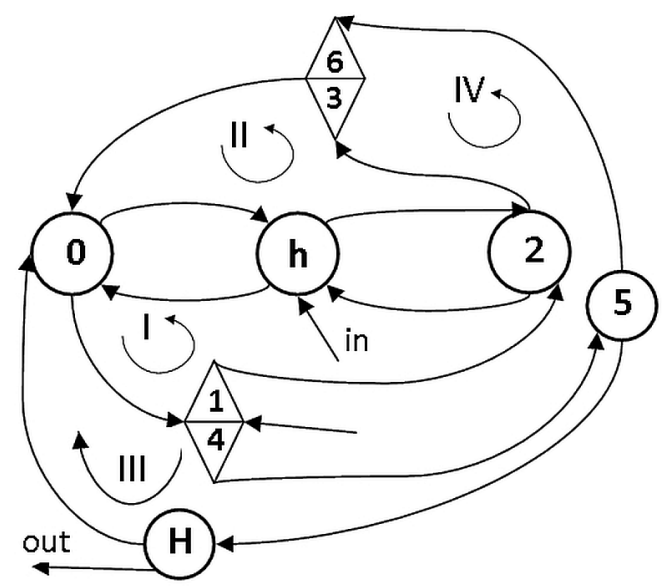

Fig. 5. Contour graph of the planetary gear.

The following contours can be distinguished for the contour graph (Fig. 5) of the considered planetary gear:

(I) $0 \rightarrow 1 \rightarrow 2 \rightarrow h \rightarrow 0$

(II) $0 \rightarrow h \rightarrow 2 \rightarrow 3 \rightarrow 0$

(III) $0 \rightarrow 4 \rightarrow 5 \rightarrow H \rightarrow 0$

(IV) $0 \rightarrow 4 \rightarrow 5 \rightarrow 6 \rightarrow 0$

Contour (I) generates the following pairs of indicators $\{(1,0) ;(2,1) ;(h, 2) ;(0, h)\}$. The contour can be interpreted as follows: we start it from 0 , i.e. the support for the axis of wheel 1 , which is geared with planet wheel 2, planet 2 rotates around its own axis mounted to $h$ and rotates together with the arm $h$ around the axis depicted by 0 . This is the end of the contour:

$0 \rightarrow 1 \rightarrow 2 \rightarrow \mathrm{h} \rightarrow 0$.

Analogous explanations can be given for the tree remaining contours. The contour graph is presented in Fig. 5 where the special drawing rules by Marghitu [20] were applied.

Based upon the distinguished contours, the following system of equations may be developed $[5,6,7,19]$ :

$$
\left\{\begin{array}{l}
\vec{\omega}_{10}+\vec{\omega}_{21}+\vec{\omega}_{h 2}+\vec{\omega}_{0 h}=0 \\
\vec{r}_{A} \times \vec{\omega}_{21}+\vec{r}_{A} \times \vec{\omega}_{h 2}=0 \\
\vec{\omega}_{h 0}+\vec{\omega}_{2 h}+\vec{\omega}_{32}+\vec{\omega}_{03}=0 \\
\vec{r}_{B} \times \vec{\omega}_{2 h}+\vec{r}_{C} \times \vec{\omega}_{32}=0 \\
\vec{\omega}_{40}+\vec{\omega}_{54}+\vec{\omega}_{65}+\vec{\omega}_{06}=0 \\
\vec{r}_{F} \times \vec{\omega}_{54}+\vec{r}_{D} \times \vec{\omega}_{65}=0 \\
\vec{\omega}_{40}+\vec{\omega}_{54}+\vec{\omega}_{H 5}+\vec{\omega}_{0 H}=0 \\
\vec{r}_{F} \times \vec{\omega}_{54}+\vec{r}_{E} \times \vec{\omega}_{H 5}=0
\end{array}\right.
$$

where $\boldsymbol{r}_{k}$ are the position vectors of the points $k=A, B, \ldots$, $F$ shown in Fig. 4 means vectors of relative angular velocity of the $i$-th element in relation to the element $j$.

$$
\left\{\begin{array}{l}
-r_{A} \omega_{1}-r_{A} \omega_{21}-r_{A} \omega_{h 2}-r_{A} \omega_{0 h}=0 \\
r_{A} \omega_{21}+r_{B} \omega_{h 2}=0 \\
-r_{C} \omega_{h 0}-r_{C} \omega_{2 h}-r_{C} \omega_{32}-r_{C} \omega_{03}=0 \\
r_{B} \omega_{2 h}+r_{C} \omega_{32}=0 \\
-r_{E} \omega_{40}-r_{E} \omega_{54}-r_{E} \omega_{H 5}-r_{E} \omega_{0 H}=0 \\
r_{F} \omega_{54}+r_{E} \omega_{H 5}=0 \\
-r_{D} \omega_{40}-r_{D} \omega_{54}-r_{D} \omega_{H 5}-r_{E} \omega_{0 H}=0 \\
r_{F} \omega_{54}+r_{E} \omega_{H 5}=0
\end{array}\right.
$$

Every contour generates two equations connected with angular velocities and some other for forces and torques. The latter are not used in the present paper. The first equation for every pair is a sum of angular velocities designated by a contour, the second one is a sum of cross products of the respective arm multiplied by the angular velocity. In the second case, some summands are omitted when the arm length is equal to zero. The following relations are used for simplification of the system [5-7, 19].

To solve the system (6-7), the following actions were undertaken: we omit the arrows, i.e. we turn the vector equations into scalar ones. It can be done because the velocities act as vectors along the same direction. Moreover, the senses (i.e. orientations along the given direction) will be established via the solution of the system. The vector multiplication (cross product) can be simplified to the scalar one, because the angles between the arms and velocities in the case of gears with cylindrical wheels are always equal to $90^{\circ}$.

For other mechanisms enclosing cranks, pistons, cylinders, sliders, followers, etc, a detailed analysis of angles has to be carried out $[5,6,7,19,20]$ to correctly simplify the system of equations. Assuming the cylindrical gear wheels, we have the relationships:

$$
r_{i}=\frac{d_{i}}{2}=\frac{z_{i} \cdot m}{2}
$$

where: $m$ is the module of the $i$-th wheel, $z_{i}-$ teeth number of the $i$-th wheel, $\mathrm{i}=1,2, \ldots, 6$.

Due to the fact that it is a universal design of multiinputs and multi-outputs, all modules were assumed as equal. It causes that the module is not present in the formulas for the output angular velocities. In the case of different modules, these formulas would be a little more complicated but the system is also solvable. The solution to system of equations is as follows:

$$
\begin{aligned}
& \omega_{6}=\omega_{3}=\frac{\omega_{H}\left(2 r_{4}+2 r_{5}\right)-r_{4} \cdot \omega_{1}}{2 r_{5}+r_{4}}=66,65 s^{-1} \\
& \omega_{h}=\frac{r_{1} \omega_{1}+\left(r_{1}+2 r_{2}\right) \omega_{3}}{2\left(r_{1}+r_{2}\right)}=84,03 s^{-1}
\end{aligned}
$$

We obtained the same numerical results as in the case of the linear graph-based modelling - see formulas. It confirms the correctness of the performed modelling and analysis. 


\section{Application of dependence graphs in tree game structures in the analysis of planetary gear}

The contour graph distribution from any vertex in the first stage leads to a tree structure with cycles, and next to a general tree game structure. Each structure has a proper analytic notation $G_{i}^{++}$- where $i$ - is a vertex, from which the graph decomposition started determining a way of transition from the dependence graph to the tree structure. In work [19], a contour graph is transformed from each vertex to a tree structure.

For example, graph distribution from vertices 0,2 leads to analytical expressions (Eq. 10-11).

$$
\begin{aligned}
& G_{p 0}^{+}={ }^{0} O^{d}[I I] \cdot h^{2}[I I] \cdot 2 c^{3}[I] \cdot h^{1},[I I \wedge I V] \cdot[6 / 3] \\
& \left.\left.\left({ }^{4}[I I] \cdot 0^{1}\right)^{4}\right)^{3},[I] \cdot 0^{2}\right)^{2},[I \wedge I I I] \cdot[1 / 4)\left([ I \wedge I V ] \cdot 2 \left([I] \cdot h^{2},\right.\right. \\
& \left.\left.\left.[\mathrm{II} / V] \cdot[6 / 3]\left({ }^{4}[I I] \cdot 0^{3}\right)^{4}\right)^{3},[I I I N I] \cdot 5\right)^{3}[I I I] \cdot H^{4}[I I I] \cdot 0^{4}\right)^{4} \text {, } \\
& \left.\left.\left.\left.[I V] \cdot[6 / 3]\left([I I] \cdot 0^{5}\right)^{4}\right)^{3}\right)^{2}\right)^{1}\right)^{0} \\
& G_{P 2}^{+}=\left(^{0} 2 l^{1}[I] \cdot h^{2}[I I] \cdot 2^{1},[I] \cdot O^{3}[I I] \cdot h^{1},[I \wedge I I I] .\right. \\
& {\left[1 / 4\left(^{4}[I \wedge V] \cdot 2^{2},[I I I \wedge I V] \cdot 5{ }^{5}[I I I] \cdot H^{6}[I I I] \cdot 0\right)\right.} \\
& \left.\left.\left.\left.\left.\left.\left.[I V] \cdot[6 / 3]\left([I I] \cdot 0^{2}\right)^{7}\right)^{6}\right)^{5}\right)^{4}\right)^{3}\right)^{2},[I I \wedge I V] \cdot[6 / 3]([I I] \cdot 0)^{2}\right)^{1}\right)^{0}
\end{aligned}
$$

Figures 6-10 show the structure from each of the vertices: $\{1 / 4\},\{6 / 3\}, 0,2,5$ of the graph (Fig. 5). In the next step, it is necessary to use complex structures.

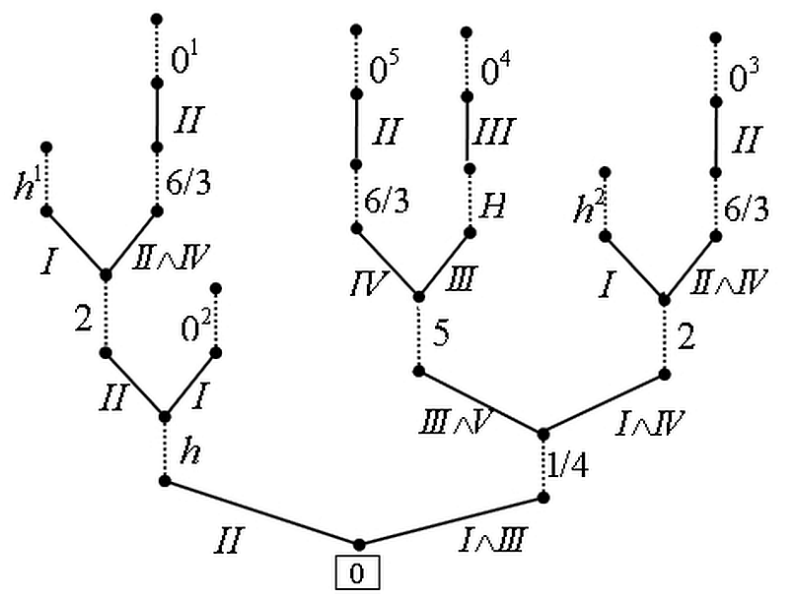

Fig. 6. Tree game structures from the initial vertex 0 .

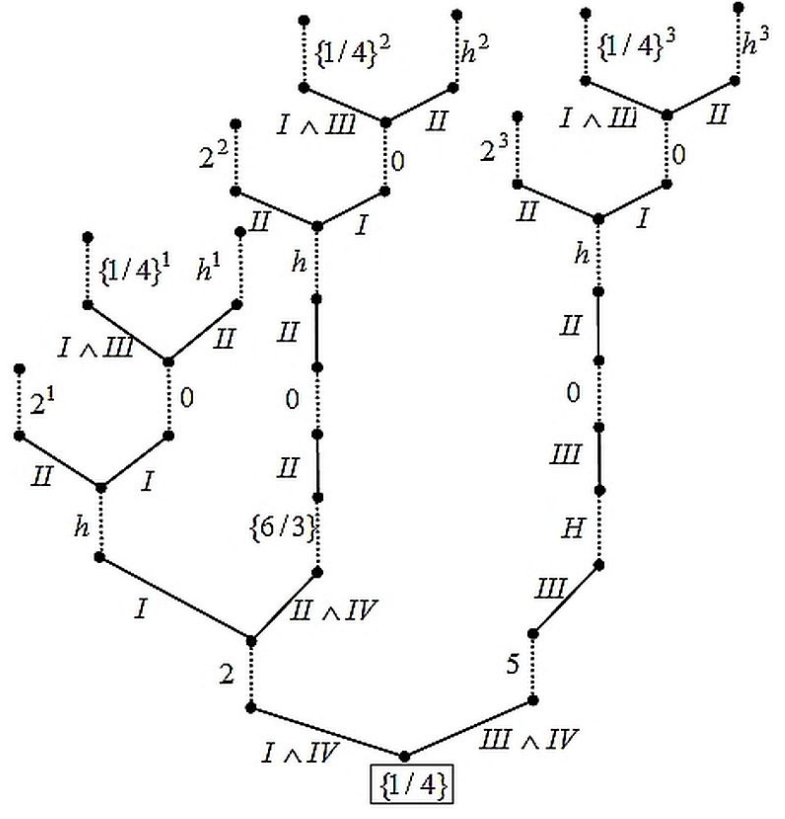

Fig. 7. Tree game structures from the initial vertex $\{1 / 4\}$.

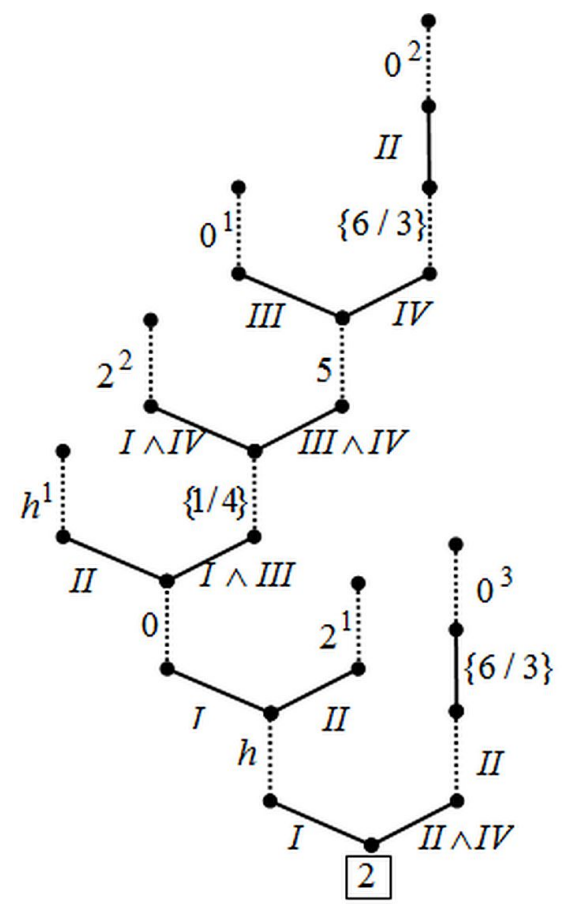

Fig. 8. Tree game structures from the initial vertex 2 . 


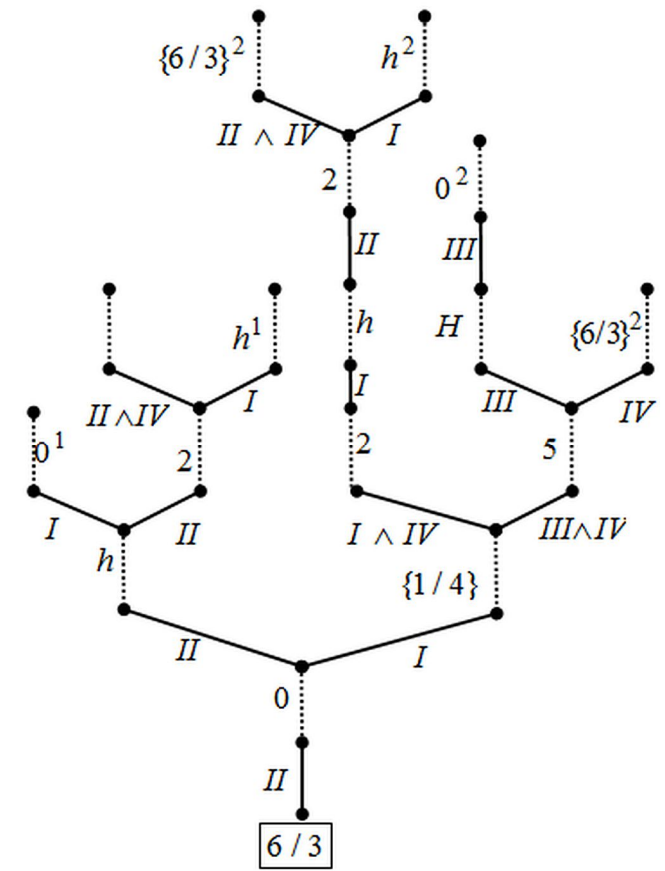

Fig. 9. Tree game structures from the initial vertex $\{6 / 3\}$.

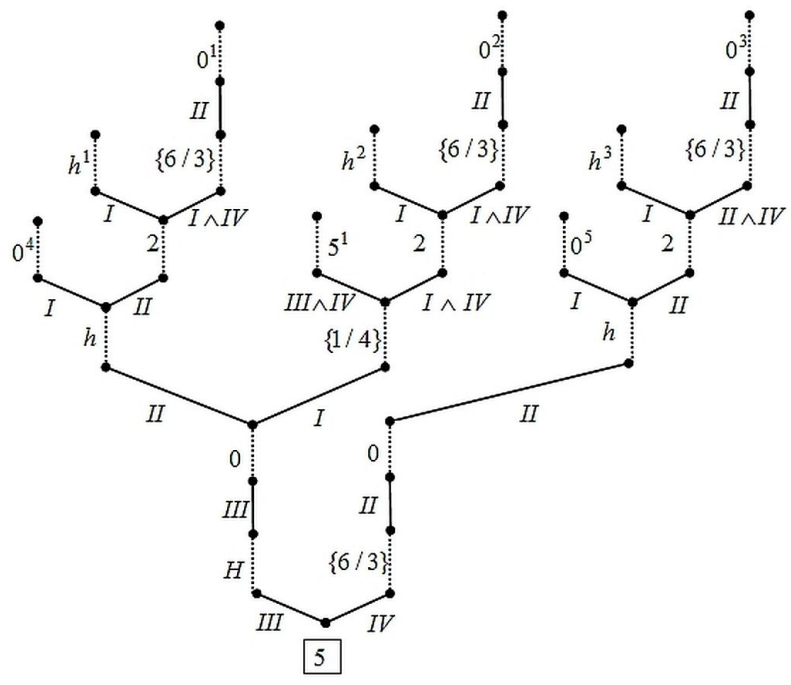

Fig. 10. Tree game structures from the initial vertex 5 .

\subsection{Application of complex parametric structures}

Complex decision trees join construction and/or operational parameters of similar properties as well as of the same adopted discretization of interval values. It makes it possible to decrease the calculation complexity in order to specify the most important subgroups of the whole set or system. In case of joining decision variables of separate properties and functions that they have in the system, a subanalysis of a given set or system is obtained [14, 19, 22, 23].

Distribution graph of the dependency of Figure 5 gives a set $\mathrm{D}$ of trees:

$$
D=\left\{G_{\{1 / 4\}}^{++}, G_{\{6 / 3\}}^{++}, G_{2}^{++}, G_{5}^{++}, G_{0}^{++}\right\}
$$

In a complex parametric tree structure, by imposing all parametric game structures, from each vertex to the game structure from the predetermined vertex, we obtain a set $S$ of total complex parametric structures:

$$
S=\left\{S_{G\{1 / 4\}}, S_{\{6 / 3\}}, S_{G 2}, S_{G 5}, S_{G 0}\right\} .
$$

Figures 11-12 show examples of complex structures: $S_{G}\{5\}$ and $S_{G\{3 / 6\}}$.

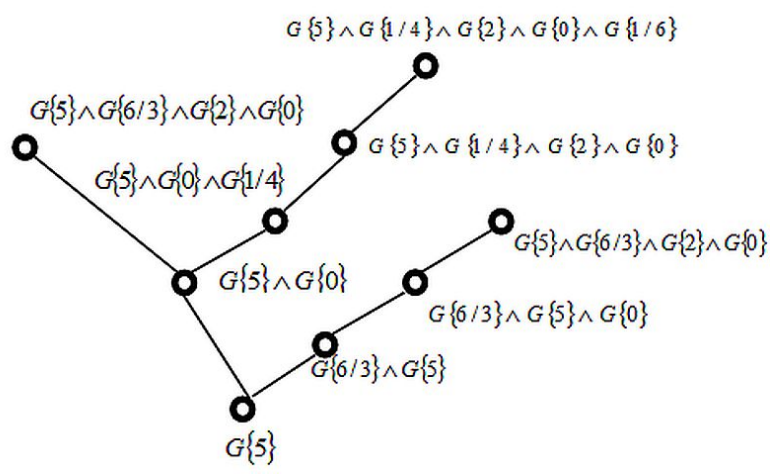

Fig. 11. Complex game structures from the initial vertex $\mathrm{G}\{5\}$.

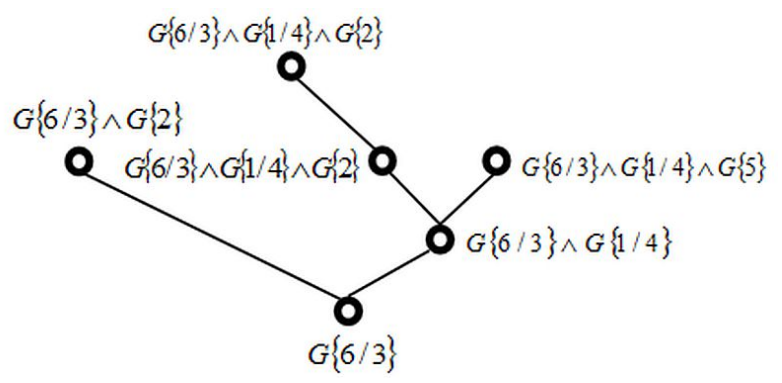

Fig. 12. Complex game structures from the initial vertex $\mathrm{G}\{6 / 3\}$.

All transformations are suitable for the contour graph of the analyzed planetary gear [22,24].

Because complex structures can be $n$ complete nodes $\gamma_{n}$, therefore, there exists a family $B$ of sets of full nodes from all the sets of the set $S$ :

$$
B \subseteq\left\{\begin{array}{l}
\left\{\gamma_{1}, \ldots, \gamma_{n}\right\}: \gamma_{1}, \ldots, \gamma_{n} \in G_{\{3 / 6\}}^{++} \wedge G_{\{1 / 4\}}^{+} \wedge G_{0}^{++} \wedge G_{2}^{++} \wedge G_{5}^{++}, \\
\gamma_{1} \neq \gamma_{2} \ldots \neq \gamma_{n}
\end{array}\right\} .
$$

Nodes on complex tree structures describe decisions, analytical-algebraic transformations and states of a given parametric play structure.

\section{Conclusions}

Thanks to the construction of parametric structures from the primary contour graph, a better representation of the planetary gear can be obtained in two areas: structural analysis and the analysis of subordinate expressions.

- Structural analysis. Each parametric game structure describes an independently grouped relational preference system. Choosing the right parametric game structure requires defining a set of variable gears to determine strategies such as solving equation systems. For a given structure, it is possible to predict whether a given 
sequence of decisions in the structure leads to the determination of the complete path of the solution of the system of equations or to find the optimal value of a given parameter.

- Analysis of subordinate expressions. An expression describing the degree of subtraction of a given component graph, i.e. the parametric game structure, is parametrically marked with a pair of parentheses within which the expression being an analytical model is written. A string formed by symbols describing vertices and edges (structural parameters and / or functions) may be subject to factorization processes, i.e. to find the product form on the basis of the product of sums or decompositions - the distribution by a set of functions. By applying dependence graphs and game tree structures we are able to present a sequence of changes of arithmetic values of constructional and service parameters in order to obtain the required behaviour of the system for the planetary gear. Unlike traditional dependence graphs and tree classifiers, the dependence graph with game tree structures implement a connection of importance rank of vertices (states) and the height of the tree structure. Application of complex game trees does not change types and graphical shapes of such structures. They become more complex but they maintain the given structural properties resulting from the initial dependence graph. Thus, a local role of decomposition can be distinguished. The introduced decomposition decision eliminates interaction of constructional and service parameters because a designer can make a decision about only single changes and observations at the successive stages. The algorithmic method for forming game structures from the contour graph describes the optimization method of systematic search. The game structure describes a space of possible solutions in order to find optimum objective functions. There is a connection with other graphical structures which can be graphs in another sense, or even decision trees with node and/or branch coding. Such interpretation can lead to different types of logical trees.

\section{References}

1. A. Deptuła, Inter. J. of Appl. Mech. and Eng. 19(3), 539-548 (2014)

2. M. A. Partyka, Algorytm Quine'a- McCluskeya minimalizacji indywidualnych czastkowych wielowartościowych funkcji logicznych (Oficyna Wydawnicza Politechniki Opolskiej, Opole, 1999)

3. S. Berghofer, M. Reiter, LNCS 5674, 147-163 (2009)

4. A. Deptuła, P. Osiński, M. A. Partyka, Arch. Civ. Mech. Eng. 13(4), 422-431 (2013)

5. S. Zawiślak, The Graph-based Methodology as an Artificial Intelligence Aid for Mechanical Engineering Design (Wydawnictwo Akademii TechnicznoHumanistycznej, Bielsko - Biała , 2010)

6. J. Drewniak, S. Zawiślak, Inter. J. of Appl. Mech. and Eng. 17, 3, 791-798 (2012)

7. J. Drewniak, S. Zawiślak, PAMM 9, 1, 547-548 (2009)

8. J. Wojnarowski, Grafy $i$ liczby strukturalne jako modele uktadów mechanicznych (Wydawnictwo Politechniki Śląskiej, Gliwice, 1986)

9. S. Uematsu, Inter. J. of the Japan Soc. For Precis. Eng. 31, 141-146 (1997)

10. J. Drewniak, S. Zawiślak, Mechanika 1, 13-21 (2010)

11. J. Drewniak, S. Zawiślak, JTAM 48, 2, 415-433 (2010)

12. L. W. Tsai, Enumeration of kinematic structures according to function (CRC Press, Boca Raton, USA, 2001)

13. C. H. Hsu, Inter. J. of Veh. Des. 13, 3, 233-241 (1992)

14. A. Deptuła, M. A. Partyka, JAMRIS 5, 3, 17-26 (2011)

15. A. Deptuła, Syst. in Manag. 2, 2, 100- 112 (2014)

16. L. Muller, Przektadnie obiegowe (PWN, Warszawa, 1983)

17. P. Y. Huang, L. H. Lu, D. Z. Chen, CSME Conf. 13, 68-73 (1996)

18. G. Kron, AIEE Trans. 49, 5, 683-685 (1930)

19. A. Deptuła, J. Drewniak, M.A. Partyka, Mechanik 7, 640- 642 (2017)

20. D. Marghitu, Kinematic chains and machine components design (San Diego. Academic Press, 2005)

21. F. Freudenstein, ASME J. of Eng. for Ind. Ser. B 93, 176-182 (1971)

22. W. Pijls, A. de B., Theo. Comp. Sci. 252, 1-2, 197-215 (2001)

23. P. P. Shwnoy, Theor. and Dec. 44, 2, 149-171 (1998)

24. Y. R. Shiue, R. S. Guh, Inter. J. of Adv. Manuf. Tech. 28, 737-746 (2006) 